Voix et Images

volxetimages

\title{
La « camera obscura » du post-modernisme
}

\section{Pierre Milot}

Volume 12, numéro 1 (34), automne 1986

Québec-Amérique latine

URI : https://id.erudit.org/iderudit/200612ar

DOI : https://doi.org/10.7202/200612ar

Aller au sommaire du numéro

Éditeur(s)

Université du Québec à Montréal

ISSN

0318-9201 (imprimé)

1705-933X (numérique)

Découvrir la revue

Citer cet article

Milot, P. (1986). La « camera obscura » du post-modernisme. Voix et Images, 12(1), 118-123. https://doi.org/10.7202/200612ar d'utilisation que vous pouvez consulter en ligne.

https://apropos.erudit.org/fr/usagers/politique-dutilisation/ 


\section{La «camera obscura» du post-modernisme}

\section{par Pierre Milot, Université du Québec à Montréal}

Les deux livres dont il sera ici question ont pour auteurs des agents qui tout en œuvrant dans des champs différents (littéraire pour l'un, philosophique pour l'autre), se rejoignent par des préoccupations intellectuelles communes. Interventions du parlogue d'André Beaudet' et l'Artiste de Pierre Bertrand ${ }^{2}$ posent une redéfinition de l'excellence esthétique en littérature et en art dans le Québec des années 1980.

Une même posture unit ces deux protagonistes de l'excellence esthétique: le sentiment profond d'être étrangers à la société et de n'y occuper aucune position, donc d'être en dehors des combines marchandes et de ne pas répondre à la demande actuelle en tant qu'écrivain et philosophe. Ces deux auteurs ont glissé si loin dans cette déclivité que l'un et l'autre risquent d'apparaître à la table des matières de la prochaine édition de l'Anthologie des poètes maudits de Pierre Seghers.

Beaudet et Bertrand se situent en rupture par rapport à l'ensemble des positions dominantes dans le champ intellectuel québécois: ils exhibent leur "solitude» et leur "singularité» face à la médiocrité ambiante. C'est le retour de Roquentin: Quelle importance ils attachent, mon Dieu, à penser tous ensemble les mêmes choses... pour exister, il faut qu'ils se mettent à plusieurs... Moi je vis seul, entièrement seul... je suis seul au milieu de ces voix joyeuses et raisonnables ${ }^{3}$. Mais dans la mesure où l'un et l'autre semblent avoir atteint ce désenchantement à leur retour de Paris (après des études doctorales), il faudrait peut-être parler du paradigme d'Ovide Plouffe. À cette différence près que l'intellectuel de la famille a vécu son drame existentiel dans l'espace social et institutionnel des années 1950.

C'est précisément ce retour du refoulé (le sujet) qui caractérise ces deux ouvrages, au-delà des différences de style (maîtrisé chez Beaudet, diffracté chez Bertrand): après la vogue structuraliste des années 60 et 70 (traduite en littérature par un formalisme où le sujet ne s'exprimait plus mais produisait des signes), le vécu reprend ses titres de noblesse et la métaphysique qu'on avait voulu "déconstruire» (le décentrement du sujet) réintroduit le «mystère". Avant d'être québécois, ce phénomène de reconversion a d'abord été le fait de l'avant-garde parisienne: Robbe-Grillet, Sarraute, Duras et Sollers ont relancé leur carrière littéraire par cette stratégie de réintégration autobiographique. Interventions du parlogue et l'Artiste répondent par conséquent à une "demande actuelle» du marché intellectuel des années 1980 , et cela malgré la dénégation formulée par Beaudet et Bertrand. En somme, ils se sont branchés sur la "camera obscura" du post-modernisme.

\section{L'hérésie consacrée ou le reliquaire d'images maudites}

Avec ce recueil de textes (publiés précédemment dans diverses revues, journaux et colloques: la Nouvelle Barre du jour, Le Devoir, Spirale, Estuaire, Colloque Pasolini, Congrès de l'Uneq etc.), And ré Beaudet poursuit 
l'opération de réinvestissement qu'il avait commencée avec la publication de Littérature, L'imposture ${ }^{4}$. Ce qui porte à penser que si Beaudet ne répond pas à "la demande", il participe considérablement à "l'offre". Pour quelqu'un qui s'assigne la prétention de déranger la gestion rationnelle du consensus moderne (p. 3), tout porte à croire que le parlogue (et son éditeur les Herbes rouges) a flairé le bon placement: car en rassemblant ces textes dont la plupart tournent autour des relations conflictuelles qu'il entretient avec les poètes de la «nouvelle écriture», Beaudet travaille plus que n'importe lequel de ses concurrents à l'institutionnalisation des enjeux suscités par ces conflits discursifs. La critique littéraire, tant journalistique qu'universitaire, n'a jamais tant parlé de la Nouvelle Barre du jour et des Herbes rouges que depuis la controverse inaugurée par le "scandale» de l'article de Beaudet ("Dialogue avec l'invisible»), paru dans Spirale en mai 1983 et portant sur une exposition de François Charron titrée Crucifixions.

Pour que l'institution littéraire puisse prendre acte, Beaudet a colligé le texte de présentation d'un livre de Charron dont il avait fait la lecture " $\mathrm{Au}$ Temporel" en 1981, et où il disait: Bizarre aventure que celle de Charron. Il s'est trompé plusieurs fois. Il a été maö̈ste, il en est revenu, il ne l'est plus... depuis ce temps-là tout va bien pour lui. On parle beaucoup de lui, on en fait l'éloge. Mais voilà, je crois que ça s'achève. Pourquoi ça s'achève? (p. 46). La réponse qu'il formule à cette question donne la mesure du coup de force symbolique anticipé (et méticuleusement embrayé) par Beaudet: On est en train de s'aviser que Charron s'est encore une fois trompé. Il est redevenu inquiétant pour la critique bien-pensante. On va se rendre compte, et vous verrez que ça ne tardera pas, que Charron est un écrivain religieux, ce qui est pire que d'avoir été marxiste (p. 46). Et pour s'assurer qu'en tant que prophète de malheur il visait juste, il ajoute une note en bas de page, datée cette fois de 1985, où il est écrit: Je ne me suis pas trompé ce soir-là, il a seulement fallu un peu plus de temps que prévu (c'est nous qui soulignons) pour que dans les têtes ça devienne clair à leur insu (p. 46). Beaudet fait ici référence à l'ensemble des textes polémiques parus dans Spirale, la Nouvelle Barre du jour et Le Devoir pour dénoncer, ou à tout le moins commenter, l'affaire. Beaudet et Charron répliqueront aux assauts des défenseurs attitrés de la modernité (copyright NBJ) par un recueil de textes publiés aux Herbes rouges (Qui a peur de l'écrivain?), et qui aura pour conséquence de rendre historique le conflit entre modernité et religiosité, tant à l'intérieur de la sphère de production restreinte (l'avant-garde) que dans l'institution littéraire. Une bonne part des Interventions du parlogue servent à consigner le procès-verbal de ce coup monté: non pas par la critique "bien-pensante» ou par les modernistes de la NBJ mais par Beaudet lui-même.

N'étant plus dans l'espace social et institutionnel de l'époque de la désespérante expérience de Borduas, époque où les scandales littéraires et artistiques étaient encore politiquement possibles, l'avant-garde des années 1980 doit aller chercher ses objets de transgression sur le marché de la postmodernité parisienne. Car la scène primitive de cette "affaire religieuse" dans le champ littéraire et culturel s'est jouée d'abord dans le Paris de la fin des années 1970 et avait pour protagonistes Bernard-Henri Lévy, auteur du Tes- 
tament de Dieu et intellectuel de promotion (au moment de la crise du marxisme français) et Philippe Sollers dont la revue emblématique, Tel Quel, devait changer son nom pour l'Infini. Ce qui peut paraître étonnant, mais cela relève de la situation objective de l'institution littéraire québécoise, c'est que l'opération symbolique ait tout de même fonctionné et qu'elle ait mobilisé un certain nombre d'agents : écrivains, éditeurs, journalistes et critiques littéraires, lecteurs (extension à un public plus élargi que la seule borne de la sphère de production restreinte qui constitue la norme de l'avant-garde en l'absence de crise). Pour réussir une telle entreprise, il fallait que Beaudet connaisse passablement bien les lois du marché littéraire et l'économie des échanges poétiques (la stratégie des concurrents). Au terme, Beaudet se débrouille fort bien dans les "combines marchandes» de la production symbolique. Mais la réussite de cette entreprise (cette percée de la «nouvelle écriture" à travers la polémique) dépend largement des rapports d'homologie structurale entre le champ littéraire québécois et le champ littéraire français, rapports qui conditionnent l'espace des styles littéraires et des stratégies de concurrence, rapports qui pèsent lourd (au sens de Max Weber) sur l'ensemble des instances de légitimation de l'institution littéraire.

Les autres "interventions du parlogue" sont articulées à partir d'un dispositif discursif où sont régulées les figures de distinction qui fondent le capital symbolique du créateur «inquiétant»: Borduas, Gauvreau, Bataille et Blanchot sont convoqués par Beaudet à témoigner du destin tragique de l'excellence esthétique. Mais à lire les deux textes dialogués où il se parle à lui-même (questions et réponses), selon les principes du genre remis à la mode par Bernard-Henri Lévy (qu'il ne cite d'ailleurs jamais), on comprend que la trajectoire et la position de Beaudet portent beaucoup moins les stigmates de Bataille ou Blanchot que la marque de Bernard-Henri Lévy: J'ai renoncé depuis longtemps à m'émouvoir de mes péchés. Et, depuis plus longtemps encore, à tenter de conjurer ces procès qui, quoi que je dise ou fasse, me sont inévitablement faits ${ }^{5}$. Quant à la façon dont il traite les figures de Borduas et de Gauvreau, il y a là une rhétorique que l'on pourrait classifier comme reliquaire d'images maudites (un recyclage du Refus global ajusté au design de la post-modernité) qui trahit la fonction tragique que Beaudet prétend assumer. Et dans la mesure où Beaudet parle de Lacan en faisant du Lacan, le moins que l'on puisse dire, en paraphrasant Lacan, c'est que c'est écrit pour que des idiots comprennent. Tout se passe comme si c'était le cas de Gérald Gaudet qui, dans un article apologétique sur Beaudet (Le Devoir, 7 décembre 85 , p. 29), laisse entendre qu'il fait partie de ceux qui comprennent: mais alors, si un critique littéraire de tendance humaniste et lyrique comme Gaudet accepte de parrainer les «interventions du parlogue», qui donc a peur d'André Beaudet?

\section{Y a-t-il un artiste dans la classe?}

Pour Pierre Bertrand, l'aune à laquelle il faut mesurer la valeur d'un "grand penseur" ou d'un "grand artiste» ne se situe pas du côté de la tradition esthétique, philosophique ou littéraire, mais dans la posture d'humilité qui caractérise les individus réellement grands de l'histoire... (qui) prennent plaisir 
à s'effacer, et même parfois à être humiliés... (p. 9). Ne recherchant ni les postes de responsabilité ni les premiers rangs, le "grand artiste» est si humble qu'aux «yeux du vulgaire» il peut paraitre avoir raté sa vie, avoir échoué dans sa vie. Destin inéluctable énonce Bertrand car les critères de la réussite sont toujours, quelque part, sociaux (p. 10). Quelque part? C'est le moins qu'on puisse dire.

Bref, pour Bertrand le monde social auquel est confrontée «la vie d'artiste" se résume à l'alternative suivante: tous ces artistes, à moins qu'ils ne deviennent célèbres (et on peut se demander à quel prix), sont considérés comme les derniers de la société, devant souvent fonctionner sur des subventions, des bourses (p. 10). D'entrée de jeu, tout pourrait porter à croire que Bertrand a produit une archéologie de l'art obscur et qu'il y a débusqué les figures stigmatisées de quelques artistes laissés pour compte par les institutions et la tradition, ou encore qu'il a rédigé un réquisitoire généreux pour les musiciens du métro et les artisans du Vieux-Montréal. Erreur d'objet, puisque le type idéal de cet artiste qui vise à passer inaperçu, à force de transparence, de sobriété... à devenir transparent à force d'obscurité (p. 11), n'est nul autre que Gilles Deleuze, auteur (avec Félix Guattari) de deux ouvrages philosophiques qui ont constitué le capital symbolique référentiel des beaux jours du post-structuralisme en France et dont on retrouve la retraduction dans les textes de la plupart des poètes formalistes québécois du milieu des années 1970 (dont André Roy, lauréat du Prix du Gouverneur général 1985): l'AntiOedipe et Mille Plateaux. Est-ce un hasard si Bertrand ne place aucun de ces poètes dans son panégyrique, à l'exception de Paul Chamberland, le seul "artiste" québécois cité nommément, pas tant pour son "œuvre» (dont rien n'est dit) mais plutôt pour sa conduite éponyme: Paul Chamberland vécut quelque chose de similaire lorsqu'il décida, bien qu'au début d'une brillante carrière, d'abandonner son poste d'enseignant à l'Université de Montréal (p. 44), et toujours selon la logique déductive de l'esthétique du vécu érigée en vertu, Bertrand surdétermine sa thèse: Et aujourd'hui, il se retrouve sans doute sans beaucoup d'argent, mais vivant comme jamais(p. 44).

Cette économie symbolique se développe ainsi sur près de deux cents pages où s'accumule le capital culturel des types idéaux (Kleist, Hölderlin, Nietzche, Kafka, Miller, Beckett) qui ne font évidemment pas partie de cette grégarité des gens qui veulent réussir à tout prix et qui prennent tous les moyens pour y parvenir (p. 11): naitre artiste, mourir artiste (selon la croyance de la main invisible). Et si, en plus, le "grand artiste" a subi un sort si misérable au point, souvent, de ne même pas parvenir à se nourrir convenablement (p. 12), c'est la béatification du cas de figure. C'est pourtant dans une salle, en train de manger (p. 70) que Bertrand a jadis fait la connaissance de l'autre auteur dont il est le débiteur le plus assidu avec Deleuze: Khrishnamurti, celui-là même dont Bertrand dit dans une formule déconcertante que pour parler de l'absence de pensée (il) doit utiliser des idées, faire appel malgré tout à la pensée de l'auditeur (p. 111). Quant à la pensée du lecteur de l'Artiste, si elle est bien "connectée» (dixit Bertrand), elle aura compris que Khrishnamurti est du genre qui ne fait pas de charme, qui passe plutôt 
inaperçu, qui est plutôt effacé (p. 72). Un Khrishnamurti qui avait anticipé le mouvement punk, mais à l'envers: Le futur n'existe que pour celui qui veut devenir quelqu'un (p. 112). Les déclassés du no future prendront acte.

Et toujours selon ce mode d'énonciation par couples d'adjectifs antagonistes qui marquent la condition sociale du "grand artiste" (gras/maigre, habillement fashion/vêtements sobres, jeune loup/brebis galeuse), Pierre Bertrand entraîne son lecteur dans les couloirs de l'Université de Vincennes, à l'époque où boursier en philosophie, il vivait comme jamais. C'est sans aucun doute le chapitre (le huitième) le plus pathétique de tout l'ouvrage, celui qui induit la position occupée par Bertrand dans le champ philosophique québécois et qui fournit les indices les plus révélateurs de la stratégie mise en œuvre dans ce livre publié dans la collection «Positions philosophiques» des Éditions de l'Hexagone. À partir de ce qu'il nomme une digression, mais qui constitue en fait la parole malheureuse et la condition de possibilité de tout l'ouvrage, Pierre Bertrand pose les points de comparaison qui opposent l'Université de Vincennes (telle qu'il l'a pratiquée) et l'Université de Montréal (telle qu'il l'a perçue à son retour de Paris): $\grave{A}$ Vincennes, il n'existait qu'un local pour tout le département de Philosophie, local défoncé, cambriolé la plupart du temps, et pour cette raison assez souvent cadenassé. Ici les professeurs disposaient de tout un secrétariat, de bureaux avec moquette. Malheureusement, ils étaient moins chanceux au niveau de ce qui finissait par ressortir de tout ce luxe (p. 74). Et toujours indélébile dans sa logique des conditions de production de la connaissance selon Jude l'obscur, il argumente: tout ce luxe avait peut-être son pendant, c'est-à-dire cette propension d'en prendre un peu trop à son aise, ou encore d'être plus ou moins acheté. Alors que du "souk» de Vincennes, quelque chose de très fort, vivant, ressortait, toute une jubilation (p. 74). Et Bertrand d'en conclure à la médiocrité innommable du champ philosophique et du système universitaire québécois.

Devant le creux barométrique d'une telle argumentation (où l'analyse institutionnelle comparative se réduit à l'ameublement des bureaux), on ne sait trop quoi rétorquer: à peine a-t-on envie de suggérer à l'auteur de l'Artiste que Deleuze a peut-être rédigé l'Anti-Oedipe dans un appartement de la rive gauche. Quant à l'assertion concernant les étudiants québécois en philosophie qui n'ont pas pu aller étudier en France et pour lesquels Bertrand s'inquiète: Par quel maître ont-ils pu être formés? Il n'en exisie aucun ici, à ma connaissance, digne de ce nom (p. 74), on peut lui répondre que ces ex-étudiants publient peut-être chez un autre éditeur et que leurs objets diffèrent peut-être du programme éditorial de la collection "Positions philosophiques" qui s'énonce comme suit: Sans être un plaidoyer pour un savoir libertaire qui s'affiche contre toute contrainte méthodologique, cette collection vise à permettre à des penseurs de réaliser des "échappées" personnelles sans avoir à se soumettre aux approbations d'un certain académisme du savoir. Qu'on pense, par exemple, à l'ouvrage collectif publié sous la direction de Claude Savary et Claude Panaccio, l'Idéologie et les stragégies de la raison: Approches théoriques, épistémologiques et anthropologiques ${ }^{6}$, et l'on comprendra qu'il y a au moins deux façons de faire de la philosophie au Québec, à des pôles institutionnels différents, et évidemment concurrentiels. 
Que Bertrand ne veuille pas se soumettre aux approbations d'un certain académisme du savoir, on le croit aisément, car il n'est pas certain que rédiger un ouvrage comme l'Artiste après que Walter Benjamin eut problématisé «la vie d'artiste» dans un livre comme Charles Baudelaire, Un poète lyrique à l'apogée du capitalisme, et après lui les héritiers de la tradition esthétique et philosophique de Francfort, Habermas et Bürger? ${ }^{7}$, soit une façon d'obtenir la note de passage... même à l'UQAM.

1. André Beaudet, Interventions du parlogue, les Herbes rouges, Montréal, 1985.

2. Pierre Bertrand, l'Artiste, l'Hexagone, collection «Positions philosophiques», Montréal, 1985.

3. Jean-Paul Sartre, la Nausée, in O.R., Gallimard, Paris, 1981, p. 11-13.

4. Voir notre critique de cet ouvrage dans Voix et images, Vol. XI, no 1, automne 1985. p. 108-113.

5. Bernard-Henri Lévy, Questions de principe, Denoël/Gonthier, collection «Médiations", Paris, 1983, p. 9.

6. Hurtubise HMH, collection «Brèches", Montréal, 1984.

7. Sur la théorie esthétique et philosophique de l'École de Francfort, voir le numéro thématique de la Revue d'Esthétique (no 8 1985), et plus particulièrement les textes d'Habermas et de Bürger.

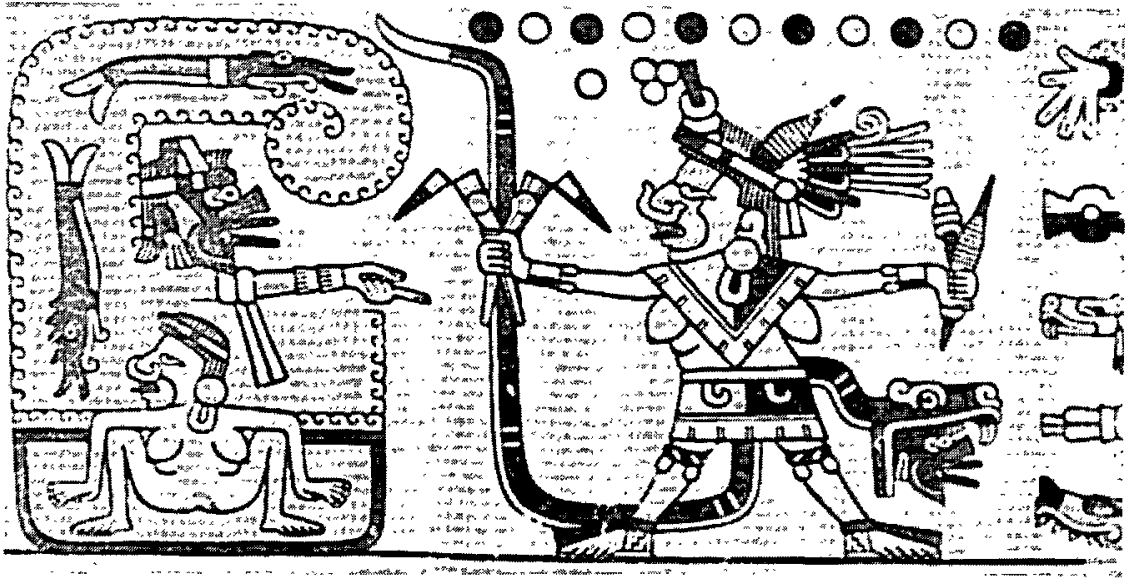

